\title{
A model of contagious bovine pleuropneumonia transmission dynamics in East Africa
}

\author{
J.C. Mariner ${ }^{\mathrm{a}, *}$, J. McDermott ${ }^{\mathrm{b}}$, J.A.P. Heesterbeek ${ }^{\mathrm{c}}$, \\ G. Thomson ${ }^{\mathrm{d}}$, S.W. Martin ${ }^{\mathrm{e}}$ \\ ${ }^{a}$ RDP Livestock Services, P.O. Box 523, 3700 AM Zeist, The Netherlands \\ ${ }^{\mathrm{b}}$ ILRI, P.O. Box 30709, Nairobi, Kenya \\ ${ }^{\mathrm{c}}$ Faculty of Veterinary Medicine, Department of Farm Animal Health, University of Utrecht, \\ Yalelaan 7, 3584 CL Utrecht, The Netherlands \\ ${ }^{\mathrm{d}}$ Epidemiology Unit, Pan African Programme for the Control of Epizootics, \\ African Union/InterAfrican Bureau for Animal Resources, P.O. Box 30786, Nairobi, Kenya \\ ${ }^{\mathrm{e}}$ Department of Population Medicine, University of Guelph, Guelph, Ont., Canada
}

Received 27 January 2005; received in revised form 21 August 2005; accepted 1 September 2005

\begin{abstract}
The dynamics of contagious bovine pleuropneumonia (CBPP) transmission vary widely between livestock production systems. This paper describes the development of a homogeneous, stochastic, compartmental model for CBPP transmission in pastoral herds of East Africa. The model was built using parameter estimates based on data published in the literature and on observations of livestock owners obtained through participatory research. The basic reproduction number for CBPP in southern Sudan was estimated to range from 3.2 to 4.6. The homogeneous model indicates that the critical community size for the persistence of CBPP falls within the typical herd sizes for pastoral communities in East Africa suggesting that individual isolated herds are capable of maintaining infection indefinitely. Vaccination alone with currently available vaccines was unlikely to eradicate the disease.

(C) 2005 Published by Elsevier B.V.
\end{abstract}

Keywords: Contagious bovine pleuropneumonia; Modelling; Basic reproduction number; Participatory; Epidemiology; Policy-making

\footnotetext{
* Corresponding author. Present address: Department of Environmental and Population Health, Tufts Cummings School of Veterinary Medicine, 200 Westboro Rd., North Grafton, MA 01536, USA.

Tel.: +1 $5088395302 / 8876762$ (direct); fax: +1 5088397948 .

E-mail address: jeffreymariner@yahoo.com (J.C. Mariner).
} 


\section{Introduction}

Contagious bovine pleuropneumonia (CBPP) caused by Mycoplasma mycoides subspecies mycoides (small colony) is a significant constraint to cattle production throughout most of sub-Saharan Africa. The disease is transmitted by direct contact and is characterized by its variable course and insidious nature. Clinical forms of the disease include the peracute, acute and chronic. Both transient, inapparent infection and the existence of persistently infected animals with encapsulated, infected sequestra are features of CBPP epidemiology. The disease is challenging to reproduce in the laboratory and study in the field. As a result, many aspects of the basic biology, epidemiology and immunology of CBPP are poorly understood (Turner, 1959; Masiga et al., 1996; Schneider et al., 1994).

Historically, CBPP was a disease of Europe, North America and Asia but was eradicated from the United States, Canada and most of Europe in the 19th century prior to the identification of the causative agent through clinical diagnosis, movement control and slaughter of suspected cases (Provost et al., 1987). Contagious bovine pleuropneumonia is believed to have been present in East and West Africa prior to the colonial era. The introduction of CBPP into southern Africa from Europe in 1854 and its subsequent spread as far north as Angola are well documented (Windsor, 2000). These regional differences are borne out by recent molecular epidemiologic studies that have demonstrated three African lineages of CBPP (Lorenzon et al., 2003).

Progress was made in controlling CBPP in Africa during the colonial era and the first two decades following independence. Large parts of Southern, Western and Eastern Africa were cleared using slaughter and movement control (Hammond and Branagan, 1965) which later incorporated testing strategies based on the complement fixation test (CFT) (Campbell and Turner, 1953; Huddart, 1960). As vaccines of moderate efficacy and duration of immunity became available, control programmes increasingly relied upon frequent vaccination and movement control.

In the 1980s and 1990s, economic crises engulfed many nations of Africa and the funding available for public veterinary services declined. Contagious bovine pleuropneumonia surveillance and control programmes were dramatically curtailed (Windsor, 2000). Public empowerment and the recognition of the pervasive negative effects of movement control on pastoral livelihoods decreased the acceptability of this tool as a control option. As a result, CBPP is again present throughout much of Eastern, Central and Western Africa (Masiga and Domenech, 1995). The East African focus has advanced south into Tanzania (Bolske et al., 1995) and subsequently spread throughout most regions of that country. The long-standing focus in Angola and northern Namibia has again invaded Zambia.

With the eradication of rinderpest from large parts of the continent, attention is returning to CBPP control. Socio-economic conditions have changed dramatically over the last 30 years, yet CBPP technology has remained essentially unchanged. Today, both movement control and test and slaughter policies would be costly to implement and poorly tolerated. Transhumant livelihoods systems are now recognized as rational and environmentally friendly foundations for sustainable development of arid lands (Niamir-Fuller, 1999). This leaves vaccination and controversial treatment regimes as the main possibilities for CBPP control in pastoral areas. 
This work combined data from the literature and livestock owner knowledge into a homogeneous model that summarizes the state of knowledge on CBPP transmission in transhumant pastoral production systems. Previously published models for CBPP were developed for sedentary animal populations using data derived from longitudinal studies (Lesnoff et al., 2004). The compartmental model described in this paper was developed for East Africa using published studies from Sudan, Kenya, Uganda, Tanzania and elsewhere in the region. Contagious bovine pleuropneumonia is readily recognized by livestock owners and traditional knowledge systems contain a wealth of information on the epidemiology of the disease (McDermott et al., 1987). A unique feature of the study was the use of data derived from the expert knowledge of pastoralists (Catley, 1999; Mariner et al., 2003). The data were collected using participatory techniques that facilitate learning from livestock owners in Sudan and Tanzania. This approach has been termed participatory epidemiology (Mariner and Paskin, 2000). Beyond factual data, participatory epidemiology identifies community priorities and interventions that are appropriate and acceptable to beneficiaries. The model allowed prediction of the impact of control measures and was used to explore control options in light of the realities of pastoral livelihoods.

\section{Materials and methods}

\subsection{Stochastic model structure}

Our stochastic compartmental model contained six principal states: susceptible, vaccinated, exposed, infectious, persistently infected and recovered (SVEIQR). The structure of the model is shown in Fig. 1. The parameter definitions and principal sources of data for their estimation are presented in Table 1.

Input parameters and the initial conditions for the exposed, infectious and resistant states were incorporated as pert probability distributions rather than discrete values. The only exception was the rate of loss of natural immunity which was entered as a constant. Pert distributions can be visualized as truncated normal distributions that lack long tails of uncharacteristic extreme values. The distributions were designed to reflect both biological variation and the uncertainty in parameter estimates. The shape of the distribution was determined by entering minimum, maximum and most likely values. In the case of the initial exposed and recovered states, a distribution was entered for the fraction immune and prevalence of exposure. This was multiplied by the population size to give the number exposed and resistant. The initial number of susceptibles was calculated from the values specified for the other states and the population size.

Latin hypercube sampling was used to select one value from each parameter distribution in each iteration of the model. Latin hypercube sampling is a two-stage stratified sampling process where each probability distribution is divided into a number of strata equal to the number of iterations to be performed. Each stratum contains an equal probability and only one value is sampled randomly from within each stratum. The strata selected for sampling for each parameter in each iteration of the simulation was chosen randomly and sampled without replacement. 


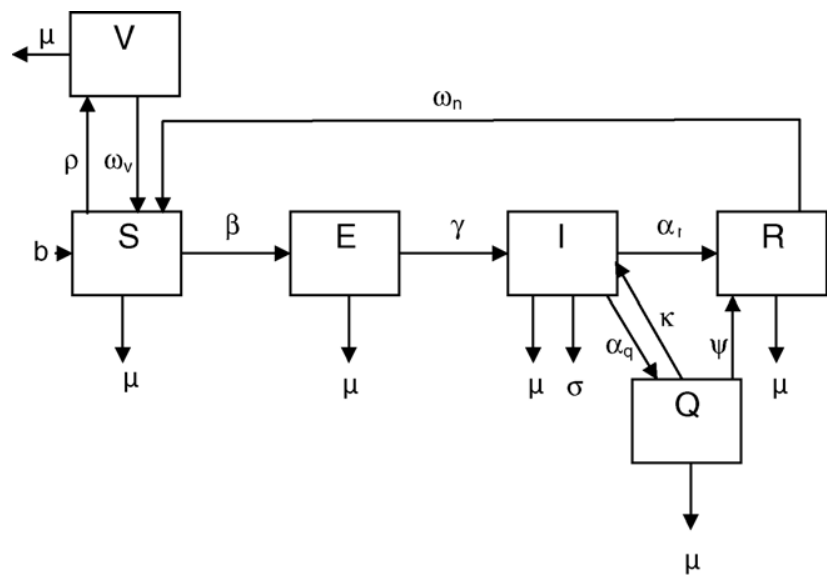

Fig. 1. Diagram of the CBPP SVEIQR model structure illustrating the relationship between the six model states and the parameters that described transition pathways. Non-specific mortality $(\mu)$ occured in all six states. Births $(b)$ all entered the susceptible $(S)$ state. Only infectious $(I)$ animals experienced CBPP mortality $(\sigma)$. The rate at which animals moved from the susceptible to exposed state $(E)$ was governed by the effective contact rate $(\beta)$. The rate at which exposed animals became infectious was described by $\gamma$. Immunization was modelled as a transition from the susceptible state directly to the vaccinal immune state $(V)$ at the immunization rate $(\rho)$. Vaccinal immunity was lost at the rate $\omega_{\mathrm{v}}$. Infectious animals could recover fully and directly enter the recovered state $(R)$ at the rate $\alpha_{\mathrm{r}}$ or develop infected sequestra $(Q)$ at the rate $\alpha_{\mathrm{q}}$. The sequestra of animals in the $Q$ state either became sterile and the individual entered the $R$ state at the rate $\psi$ or were re-activated at the rate $\kappa$ and the individual reentered the infectious state. Animals in the $R$ state were naturally immune but may possessed sterile sequestra. Naturally immune animals could lose their immunity at the rate $\omega_{\mathrm{n}}$ and return to the susceptible state.

Table 1

Minimum, mode and maximum estimates of used in the pert distributions for the parameters of the SVEIQR homogeneous population model $\left(\right.$ days $^{-1}$ )

\begin{tabular}{|c|c|c|c|c|c|}
\hline & Definition & Min & Mode & Max & Key information sources \\
\hline$\alpha_{\mathrm{q}}$ & Rate of sequestrum formation & 0.011 & 0.013 & 0.018 & $\begin{array}{l}\text { Gourlay, 1965; Bygrave et al., 1968; } \\
\text { Turner and Etheridge, } 1963\end{array}$ \\
\hline$\alpha_{\mathrm{r}}$ & Rate of recovery & 0.0036 & 0.0045 & 0.0059 & Parker, 1960; Stone and Bygrave, 1969 \\
\hline$\beta$ & Effective contact rate $(\mathrm{Cp})$ & 0.07 & 0.126 & 0.13 & Mariner et al., 2003 \\
\hline$\gamma$ & $\begin{array}{l}\text { Transition rate from exposed } \\
\text { to infectious state }\end{array}$ & 0.018 & 0.024 & 0.036 & $\begin{array}{l}\text { Hudson and Turner, 1963; Turner and } \\
\text { Campbell, 1937; Bygrave et al., } 1968\end{array}$ \\
\hline$\kappa$ & Rate of sequestrum reactivation & 0.00007 & 0.00009 & 0.00011 & $\begin{array}{l}\text { Bygrave et al., 1968; Windsor } \\
\text { and Masiga, } 1977\end{array}$ \\
\hline$\mu$ & Non-specific mortality rate & 0.00050 & 0.00055 & 0.00062 & Zessin et al., 1985; Majok et al., 1991 \\
\hline$\rho$ & $\begin{array}{l}\text { Rate of immunization } \\
\text { (successful vaccination) }\end{array}$ & - & - & - & User defined \\
\hline$\sigma$ & CBPP-specific mortality rate & 0.0064 & 0.0090 & 0.013 & Mariner et al., 2003 \\
\hline$\omega_{\mathrm{n}}$ & Rate of loss of natural immunity & - & 0.00027 & - & $\begin{array}{l}\text { Constant (Mariner et al., 2003; } \\
\text { Windsor and Masiga, 1977) }\end{array}$ \\
\hline$\omega_{\mathrm{v}}$ & Rate of loss of vaccinal immunity & 0.00078 & 0.00091 & 0.0011 & $\begin{array}{l}\text { Wesonga and Thiaucourt, } 2000 \text {; } \\
\text { Gilbert et al., 1970; Windsor et al., } 1972\end{array}$ \\
\hline$\psi$ & Rate of sequestrum resolution & 0.0068 & 0.0075 & 0.0079 & Bygrave et al., 1968 \\
\hline
\end{tabular}


The model utilized an open population design where animals are born into the susceptible $(S)$ state at each time step at rate $b$. Non-specific mortality occurred at the rate $\mu$ and resulted in losses from all six states. The non-specific mortality rate and the estimated birth rate were set equal. Contagious bovine pleuropneumonia specific mortality occurred at the rate $\sigma$ from the infectious $(I)$ state. In surviving animals, the lesions either healed and the individuals entered directly into the recovered $(R)$ state or the lesions resolved through a process of sequestration. Sequestra were considered to be encapsulated and infected, but not infectious (Turner, 1954). Animals that developed infected sequestra entered the persistently infected $(Q)$ state. As sequestra resolved and/or became non-infected, the animals transitioned to the recovered $(R)$ state. Note that animals in the recovered state could have sterile sequestra. Alternately, infected sequestra could occasionally be reactivated and in this instance the animal would transition from the persistently infected $(Q)$ state back to the infectious $(I)$ state.

We assumed random mixing of all individuals in the population. The mass action term incorporated the population size $(N)$ at each time step as the sum of the numbers in the six states $(S+V+E+I+Q+R)$.

The difference equations were:

$$
\begin{aligned}
& \Delta S=-C p \frac{S I}{N}(\Delta T)+b N(\Delta T)+\omega_{\mathrm{v}} V(\Delta T)+\omega_{\mathrm{n}} R(\Delta T)-\rho S(\Delta T)-\mu S(\Delta T) \\
& \Delta V=\rho S(\Delta T)-\omega_{\mathrm{v}} V(\Delta T)-\mu V(\Delta T) \\
& \Delta E=C p \frac{S I}{N}(\Delta T)-\gamma E(\Delta T)-\mu E(\Delta T) \\
& \Delta I=\gamma E(\Delta T)+\kappa Q(\Delta T)-\alpha_{\mathrm{r}} I(\Delta T)-\alpha_{\mathrm{q}} I(\Delta T)-\sigma I(\Delta T)-\mu I(\Delta T) \\
& \Delta Q=\alpha_{\mathrm{q}} I(\Delta T)-\kappa Q(\Delta T)-\psi Q(\Delta T)-\mu Q(\Delta T) \\
& \Delta R=\alpha_{\mathrm{r}} I(\Delta T)+\psi Q(\Delta T)-\omega_{\mathrm{n}} R(\Delta T)-\mu R(\Delta T)
\end{aligned}
$$

We will now describe the meaning of the parameter choices made.

The number of transitions from the susceptible to the exposed state per time step was calculated using:

$$
\beta \frac{S I}{N}
$$

where the effective contact rate, $\beta$, is equal to the product of the physical contact rate $(C)$ and the probability of transmission per contact $(p)$. Seasonal fluctuations in contact rates were modelled by a periodic trigonometric function to adjust the values of $\beta$ (Grenfell et al., 1995). This was incorporated in the model as:

$$
\beta(t)=\beta_{0}[1+A \sin (2 \pi t)]
$$

where $\beta_{0}$ is the average effective contact rate and $A$ controls the amplitude of the seasonal variation. The model was constructed so that $A$ could be adjusted by changing a single cell value. The intensity of this effect could be varied between no effect and full seasonality by entering a fraction between 0 and 1 . 
The recovery rate $(\alpha)$ consisted of two components. The rate at which animals moved from the infectious to the recovered state was $\alpha_{\mathrm{r}}$. Sequestration, an indirect route to full recovery, was described in the model by $\alpha_{\mathrm{q}}$. The average duration of the infectious period was therefore given by:

$$
\frac{1}{\mu+\alpha_{\mathrm{r}}+\alpha_{\mathrm{q}}+\sigma}
$$

where $\sigma$ was the CBPP-specific mortality rate and $\mu$ the non-specific mortality rate.

Once animals entered the persistently infected state they could either recover fully at the rate $\psi$, or the infection could reactivate with relatively low probability and the animals reenter the infectious state as described by the rate $\kappa$. The average duration of the persistently infected state was therefore given by:

$$
\frac{1}{\psi+\kappa+\mu}
$$

where $\kappa$ and $\mu$ were small relative to $\psi$.

Vaccinal immunity was modelled as a separate state reflecting the duration and quality of immunity due to vaccination. The proportion of susceptibles immunized, $\rho$, was determined by the proportion vaccinated, $p_{\mathrm{v}}$, the proportion of vaccinations properly applied (efficiency of vaccination), $p_{\mathrm{e}}$, and the efficacy of the vaccine, $\varepsilon$, according to the formula:

$$
\rho=p_{\mathrm{v}} p_{\mathrm{e}} \varepsilon
$$

All three sub-parameters could be adjusted in the model. The parameters $p_{\mathrm{e}}$ and $\varepsilon$ were set globally, whereas the proportion vaccinated and the time interval between vaccinations could be set for each round of vaccination. All vaccination in each round was pulsed in one time step. The rate at which animals lost vaccinal immunity and returned to the susceptible state was $\omega_{\mathrm{v}}$. Animals that recovered from natural infection could lose immunity and return to the susceptible state at a much lower rate, $\omega_{\mathrm{n}}$.

Apart from the probability distributions for the values of the major parameters, the model incorporated two stochastic elements. First, as only whole animals can transit between states, a stochastic form of rounding was used for all transitions. The fractional portion of transitions was compared against a random number to determine the direction of rounding. The second stochastic element determined the number of transitions from the susceptible $(S)$ to the exposed $(E)$ compartment when the predicted number of transitions was below a user-defined stochastic threshold (e.g. five or less). When the number of transitions calculated by Eq. (7) was less than the stochastic threshold, the result was compared against a random number and either a number of transitions equal to the stochastic threshold or no transitions took place during the time step. The stochastic effects regulate the timing of transitions and cause them to occur in discrete packets or not at all. The stochastic effects did not change the total number of transitions expected to occur in an iteration of the model, provided these chance effects did not lead to fade-out of the disease from the population. 


\subsection{Parameter estimation}

\subsubsection{Duration of exposed states}

As data on the onset of shedding of infectious material by infected animals are not available, the latent period $(1 / \gamma)$ was estimated from information on the incubation period available in the literature. These studies primarily measured the time between documented exposure and the detection of complement-fixing antibodies. The incubation period for CBPP has been reported to range between 20 and 123 days (Hudson and Turner, 1963). However, $91.4 \%$ had sero-converted by day 60 and only one animal had an incubation period greater than 70 days. Turner and Campbell (1937) described a narrower incubation period of 29-58 days. Bygrave et al. (1968) provided limited data to indicate an incubation period of $31-45$ days.

\subsubsection{Duration of acute and persistent infections}

The model structure assumes that exposed $(E)$ cattle become infectious $(I)$ and then either recover fully $(R)$ or enter the non-infectious but persistently infected state $(Q)$. Different measurements and observations from the literature can be used to estimate the duration of the infectious and persistently infected states, either singly or combined. The herders reported that clinical disease persisted for up to 12 months with an average duration of 3.9 months (Mariner et al., 2003). From the literature, field investigations supported by CFT testing and post mortem examinations described the average duration of active disease as approximately 6 months (Parker, 1960; Stone and Bygrave, 1969). A mean duration of infection (combined length of infectious and persistently infected states) of 6 months (27 weeks) with a range of 24-31 weeks was taken to reflect the range of clinical and laboratory experience. This was subsequently split into the infectious and persistently infected periods.

As the experimental and field observations concerned survivors only both $\mu$ and $\sigma$ were dropped from Eq. (9) for parameter estimation. This allowed the quantity $1 /\left(\alpha_{\mathrm{r}}+\alpha_{\mathrm{q}}\right)$ to be estimated from the duration of the infectious period. Similarly $\mu$ could be dropped from Eq. (10) and as the rate of re-activation $(\kappa)$ is small, the relationship between the duration of the persistently infected state and the rate of sequestrum resolution was simplified to $1 / \psi$ for the purposes of parameter estimation.

The relative percentage of animals that recover directly versus those that pass through the persistently infected state was estimated in order to divide $\alpha$ into its two component rates. Several papers have assessed the prevalence of sequestra in recovering CFT reactors in pastoral outbreaks in East Africa. The observations of Turner and Etheridge (1963) and Bygrave et al. (1968) indicated that the majority of survivors develop sequestra. With the exception of one study that found only 1 of 80 survivors developed a sequestra (Parker, 1960), other studies reported between 56.6 and $76.1 \%$ of survivor developed sequestra (Gourlay, 1965; Davies et al., 1968; Stone and Bygrave, 1969; Gilbert et al., 1970; Windsor et al., 1972).

Bygrave et al. (1968) published a very detailed analysis of the lesions encountered in a pastoral outbreak of CBPP in Northeastern Kenya. The relative frequency of acute, subacute (early sequestra) and chronic (sequestra) lesions suggest that in excess of $80 \%$ of survivors develop sequestra. In the model, it was estimated that $75 \%$ of surviving clinical 
cases recovered through a process of sequestrum formation and the overall recovery rate was divided into its two component rates using $3 \alpha_{\mathrm{r}}=\alpha_{\mathrm{q}}$.

The number of animals in the persistently infected state was determined primarily by the rate of sequestra formation $\alpha_{\mathrm{q}}$ and the rate of sequestra resolution $\psi$. Bygrave et al. (1968) showed that sequestra accumulated in the population as the outbreak progressed. When the outbreak had subsided at week 16 , there were roughly six times as many sequestra as acute cases. This pattern was used to partially validate the model.

The final step was to estimate $\kappa$, the rate of sequestra re-activation. Bygrave et al. (1968) found only two cases of early CBPP lesions in combination with sequestra out of 2544 post mortem examinations. Of these 2544, the authors reported that 582 had sequestra. The low incidence of possible reactivation reported by Bygrave et al. (1968) agrees with Windsor and Masiga (1977) in their inability to reactivate sequestra using a variety of stresses. Considering that the acute lesions persist no more than 1 month relative to the 4 month persistently infected period, the Bygrave et al. result suggested that two sequestra reactived per month over the 4-month life of sequestra (4 months $\times 2$ reactivations per month per 582 cases) or that $1.4 \%$ of sequestra reactivate.

\subsubsection{The transmission parameters $\left(\beta\right.$ and $\left.R_{0}\right)$}

The product of $C$ and $p$ is the effective contact rate $(\beta)$. All three of these quantities are in practice very difficult to measure. However, $\beta$ can be derived from the basic reproduction number which can be estimated from serological data. The basic reproduction number $R_{0}$ is the number of secondary infections resulting from one primary case in a totally susceptible population (Anderson and May, 1991; Diekmann and Heesterbeek, 2000). The basic reproduction number is a feature of both the infectious agent and the host population without a control measure being active. Taking into account the persistently infected state, the formula for $R_{0}$ for model $1-6$ can be written as:

$$
R_{0}=\beta \frac{\gamma}{(\gamma+\mu)} \frac{\kappa+\psi+\mu}{\left(\mu+\alpha_{\mathrm{r}}+\alpha_{\mathrm{q}}+\sigma\right)(\kappa+\psi+\mu)-\alpha_{\mathrm{q}} \kappa}
$$

As the value of $\kappa$ approaches zero, the formula for $R_{0}$ can be reduced to:

$$
R_{0}=\beta \frac{\gamma}{(\gamma+\mu)\left(\mu+\alpha_{\mathrm{r}}+\alpha_{\mathrm{q}}+\sigma\right)}
$$

where $\gamma /(\gamma+\mu)$ is the probability that a newly infected individual survives the latent period and where $1 /\left(\mu+\alpha_{\mathrm{r}}+\alpha_{\mathrm{q}}+\sigma\right)$ is the average duration of the infectious period, during which $\beta$ new cases occur per unit of time. Thus, $\beta$ can be estimated from Eq. (13) using values for $R_{0}$, the principal model parameter estimates described in Sections 2.2.1 and 2.2.2, the specific mortality rate based on livestock owner reports (Mariner et al., 2003) and the non-specific mortality rate (Zessin et al., 1985; Majok et al., 1991). It is important to bear in mind that the estimated values of $R_{0}$ are not directly entered into the model. They are used to estimate the distribution for $\beta$ that is input to the model.

In populations where the force of infection is constant and vaccination is not practiced, the value of $R_{0}$ can be estimated deterministically from serological measures of the average age of infection (Dietz, 1975). The appropriate formula for a population with an 
approximately constant mortality rate across all ages is:

$$
R_{0}=(L / A)+1
$$

where $L$ is the average life expectancy and $A$ the average age at infection.

The basic reproduction number for CBPP was estimated using data from two published serological studies (McDermott et al., 1987; Zessin et al., 1985) conducted in Dinka and Fulani cattle populations and from an age-structured sample of Jie, Murle and Dinka cattle collected as part of the present project (Mariner et al., 2003). The data from the literature were based on the CFT test (Campbell and Turner, 1953), whereas Mariner et al. (2003) utilized both the CFT and C-ELISA test (Le Goff and Thiaucourt, 1998). All populations were unvaccinated. Complement fixation reactions are believed to persist for 6-12 months regardless of the clinical state of the test subject. It was assumed that the mean duration of CFT antibody was 9 months which implies that on average a seropositive animal was infected 4.5 months previously. For positive animals, the age of infection was calculated as the median of the age category at testing less 4.5 months. The estimated $R_{0}$ values were adjusted for the season of collection to provide a baseline annual estimate for incorporation in the seasonally forced model.

\subsubsection{Vaccine efficacy and duration of immunity}

Extensive literature on the efficacy of the currently recommended T1/44 vaccine strain is available. All papers used a complex qualitative, non-parametric scoring method developed by Hudson and Turner (1963) where the score for indicators of disease and infection in vaccinates is divided by the score in controls and the result was subtracted from 1. The scores do not directly assess the impact of vaccination on the ability of vaccinates to shed or transmit infection.

A review of the T1/44 vaccine strain efficacy trials found seven papers that had evaluated the vaccine at 3-6 months post vaccination (Davies et al., 1968; Gilbert et al., 1970; Karst, 1971; Masiga and Read, 1972; Masiga et al., 1978; Wesonga and Thiaucourt, 2000; Thiaucourt et al., 2000). The reported efficacy scores for protection against macroscopic pathologic lesions $\left(E_{\mathrm{p}}\right)$ ranged between 33 and $95 \%$. Three articles reported $E_{\mathrm{p}}$ values between 66 and $75 \%$ from challenge experiments conducted at 12-15 months post vaccination (Gilbert et al., 1970; Masiga et al., 1978; Wesonga and Thiaucourt, 2000). Finally, Windsor et al. (1972) found an $E_{\mathrm{p}}$ of $80 \%$ for the T1/44 strain in cattle challenged 2 years post vaccination, although 5 of 16 vaccinates $(31.3 \%)$ developed infected sequestra as a result of in-contact challenge.

\subsection{Stochastic model experimentation}

The input parameter estimates are summarized in Table 1 as rates per day. These values were used in all simulations unless otherwise noted. When the rates are re-stated as periods, a mean duration of 6 weeks and range of 4-8 weeks was used as the latent period. The acute phase or infectious period lasted 6-10 weeks followed by a persistently infected, but noninfectious, sequestrum phase of longer duration. Based on an average period of infection of 27 weeks, the average persistently infected period was estimated to be 19 weeks $(0.0075$ resolutions per sequestrum-day) with a range of 18-21 weeks. The estimate of the average 
duration of sequestra $\kappa$ was 19 weeks $(0.012 / 133$ days or 0.00009 activations per sequestrum-day).

The efficacy of the vaccine in terms of the interruption of transmission was estimated to be $65 \%$ with a range of $50-80 \%$. The duration of immunity $\left(1 / \omega_{\mathrm{v}}\right)$ was estimated as 3 years. The proportion vaccinated, $p_{\mathrm{v}}$, and the efficiency of vaccination $p_{\mathrm{e}}$ were set to 0.8 in all instances based on the realistic performance of mass vaccination teams as assessed by the Pan African Rinderpest Campaign (IAEA, 1992; IAEA, 1993).

The deterministic estimates of $R_{0}$ (Table 2) ranged between 3.2 and 4.8 with a most likely value of 4.1 . As a result, $\beta$ ranged between 0.07 and 0.13 with a most likely value of 0.126 .

Quantitative outputs from the model were probability distributions and included $R_{0}$, the duration of infection in the population, the prevalence of infection at the end of the run, the overall case fatality rate and the total number of cases, deaths and vaccinations. The distribution of $R_{0}$ values output by the model was compared against the values of $R_{0}$ estimated serologically that were used in the estimation of $\beta$, as an internal check on consistency. Averages of point prevalence of infective and persistently infected individuals as well as the immunity proportion were calculated over 6-monthly intervals. The model produced graphic data including epidemic curves that provide information on the pattern and periodicity of epidemics.

Approximate values for the initial prevalence of immunity and exposure in endemic settings were determined by setting the initial population size to 10,000 head to eliminate stochastic fade-outs, setting the amplitude of the seasonal forcing to 0 and observing the final states. The stochastic comparison was set to 5 . The inter-epidemic period in the absence of seasonal forcing was determined from the same model run.

Sensitivity analysis was completed using linear regression and coefficients are reported as standardized values between -1 and +1 .

A population size of 500 and amplitude of seasonal forcing of 0.5 were used in all subsequent simulations unless otherwise noted. Initial conditions for the exposed and

Table 2

Estimates of $R_{0}$ for the field transmission of CBPP calculated from serological data and used in the estimation of $\beta$ and internal validation of the SVEIQR homogeneous population model

\begin{tabular}{llllllll}
\hline Community or location & Year $^{\mathrm{a}}$ & $A^{\mathrm{b}}$ & $L^{\mathrm{c}}$ & $\operatorname{Adj}^{\mathrm{d}}$ & $R_{\mathrm{o}}$ & $h^{\mathrm{e}}(\%)$ & Source \\
\hline Dinka, Sudan & 1980 & 1.5 & 4.5 & 0.1 & 4.8 & 79 & Zessin et al., 1985 \\
Fulani, Sudan & 1980 & 1.8 & 4.5 & 0.1 & 4.2 & 76 & Zessin et al., 1985 \\
Tuic Dinka, Sudan & 1984 & 2.9 & 5.0 & 0.25 & 3.4 & 71 & McDermott et al., 1987 \\
Boma, Sudan & 2002 & 3.2 & 4.9 & 0.25 & 3.2 & 69 & Mariner et al., 2003 \\
Dinka, Sudan $^{\mathrm{f}}$ & - & - & - & - & 4.5 & 78 & Mefit-Babtie, 1983 \\
Australia $^{\mathrm{g}}$ & - & - & - & - & 4.0 & 75 & Turner, 1954 \\
\hline
\end{tabular}

\footnotetext{
a Year of survey.

b Average age of infection in years.

c Life expectancy.

d Seasonal adjustment for period of sample collection.

e The herd immunity threshold required to interrupt transmission.

${ }^{f}$ Based on number of secondary cases.

$g$ Based on end point of outbreak.
} 
infectious states were set to a prevalence range of $0.01-0.03$ with a most likely value of 0.02. A prevalence range of $0.50-0.60$ with a most likely value of 0.55 was used to calculate the initial number in the recovered state. The initial numbers vaccinated and persistently infected were set to 0 .

The impact of the size of the stochastic comparison used to determine the number of transitions from the susceptible to exposed state and the presence or absence of seasonal forcing on the persistence of CBPP in herds of various sizes was studied in a series of model runs. Stochastic comparisons of 0 or 5 were compared in herds ranging between 50 and 1000 head. The amplitude of the seasonal forcing function was either set to 0 or 0.5 .

The impact of decreasing the infectious period on the persistence of CBPP in herds of 500 head was investigated by setting the overall value of $1 / \alpha$ to most likely values of 56,42 and 28 days in successive simulations while maintaining the ratio between $\alpha_{\mathrm{r}}$ and $\alpha_{\mathrm{q}}$ constant.

The impact of mass immunization on the persistence of infection in individual herds (herd level prevalence) was assessed under three different scenarios: annual campaigns over 5 years, biannual campaigns over 2 years and biannual campaigns over 3 years. Two ranges of vaccine efficacy were evaluated: $50-80 \%$ efficacy and $60-80 \%$ efficacy. The duration of immunity due to vaccination was set to a range of $2.75-3.25$ years with a most likely value of 3.0 years.

\subsection{Model validation}

Participatory epidemiologic techniques were used to collect data on case mortality, inter-epidemic period, seasonality, and the clinical course of infection from livestock owners of the Jie, Murle, Dinka and Nuer pastoral communities of southern Sudan and the Tatuga and Kurya communities of northern Tanzania (Mariner et al., 2003). The model was validated in part by its ability to reproduce livestock owner expert opinion. The epidemic curves for infectious and chronic cases produced by the model were also compared to the data generated by Bygrave et al. (1968) from north-eastern Kenya.

Model predictions of the prevalence of infection (infectives and persistently infected) and $R_{0}$ were compared against estimates derived from published serological surveys and sample sets collected as part of this study.

\section{Results}

A histogram of the distribution of $R_{0}$ values generated by the model in the initial run of 500 iterations and a population size of 10,000 is presented in Fig. 2. Note that $90 \%$ of the values lay between 3.4 and 4.7 with a mean of 4.1 . The distribution was left skewed. The distribution of average prevalences of infected animals during the final 6 months of the iterations is presented in Fig. 3. Ninety percent of the iterations ended with average prevalences between 4.3 and $7.4 \%$. A sensitivity analysis to estimate the impact of all input parameters on the total number of cases is presented in Fig. 4. The total number of cases was inversely related to the CBPP-specific mortality rate, $\sigma$, and by positive relationships 


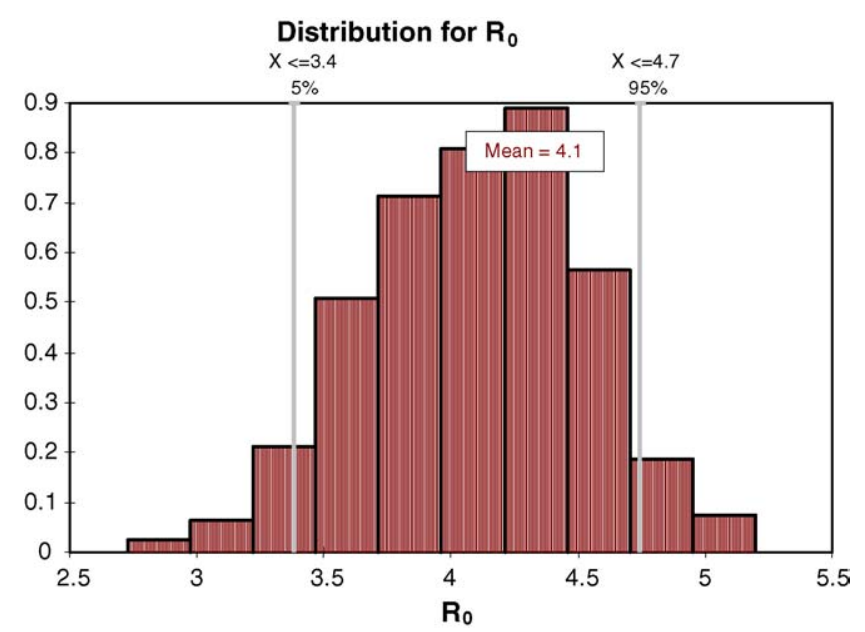

Fig. 2. A histogram of $R_{0}$ values representative of a typical model run of 500 iterations. Note that $90 \%$ of the values lay between 3.38 and 4.74 . The distribution was skewed slightly to the left which is consistent with the fact that most of the empirical estimates made from field data were greater than 4 .

with the effective contact rate, $\beta$, and the non-specific mortality rate, $\mu$. The regression model for total cases had an $r^{2}$ of 0.654 . The persistence of infection was also highly sensitive to $\sigma(-0.803)$ and moderately sensitive to $\alpha_{\mathrm{q}}(0.234), \beta(0.091)$ and $\gamma(0.072)$ with an $r^{2}$ of 0.704 .

When the herd size was reduced to 500 head, the sensitivity profile changed. Total cases was sensitive to $\gamma(-0.175), \beta(0.149), \alpha_{\mathrm{r}}(-0.145)$ and $\sigma(-0.129)$ but the $r^{2}$ of the regression model was only 0.100 . The persistence of infection was sensitive to $\gamma(-0.164)$ and $\psi(0.108)$ with an $r^{2}$ of only 0.040 .

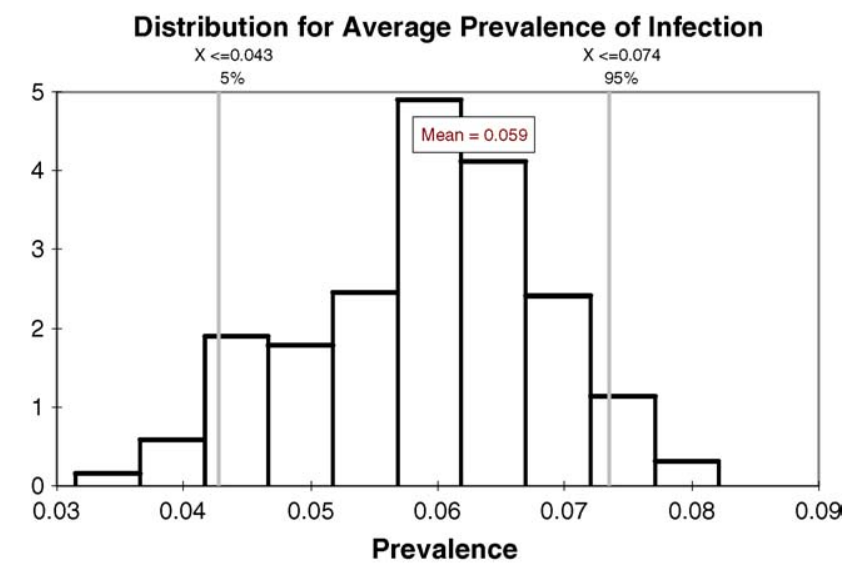

Fig. 3. The average animal level prevalence of infection (infectives and persistently infected sequestra) had a range of 3.2-8.2\% during the final 6 months of the model runs. Ninety percent of the prevalence predictions fell between 4.3 and $7.4 \%$. 


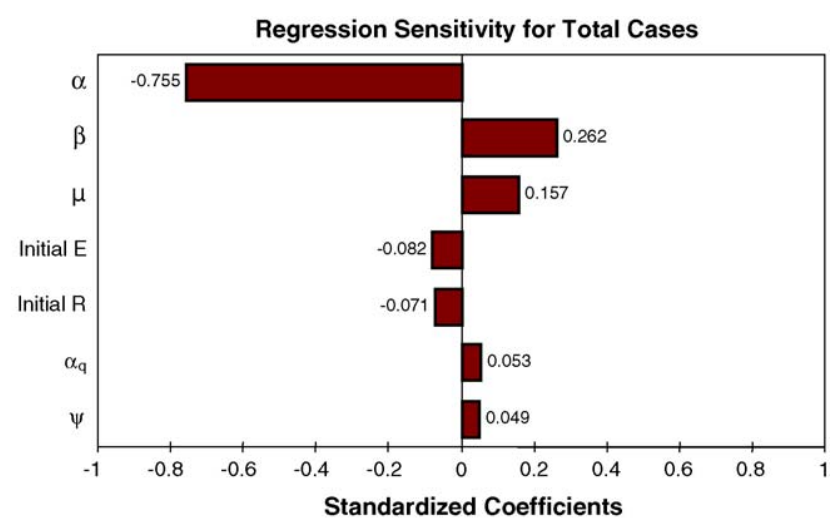

Fig. 4. The sensitivity of the total number of cases to input parameters is shown in this tornado plot. Note that the total number of cases was most sensitive to the CBPP mortality rate, $\sigma$, followed by the effective contact rate, $\beta$. Initial $E$ and initial $R$ were the initial number exposed and the initial number recovered, respectively. These initial conditions exerted a minor effect on the total number of cases predicted by the simulation.

The predicted average of the daily prevalence for the final 6 months of the model run for infectious cases, persistently infected and recovered immune animals is presented in Table 3. The predicted range and mean value for the inter-epidemic period in the absence of seasonal forcing is also presented.

The results of trials to determine the impact of varying seasonality and the stochastic comparison for transitions between the susceptible and exposed state in herd sizes of 50 1000 head are presented in Table 4. Note that when the stochastic comparison is set to 5 and seasonal forcing with amplitude of 0.5 is applied, disease fades out in essentially all herds of 300 head or less. If seasonal forcing is eliminated, disease occasionally persists in herd sizes between 75 and 300 . When the stochastic comparison for animals transitioning to the exposed state is set to 0 , the model predicts that CBPP can persist in herds of 100 head in $2 \%$ of cases.

The impact of decreasing the infectious period on CBPP persistence and overall mortality in herds of 500 head is presented in Table 5. Note that decreasing the infectious period caused a reduction in $R_{0}$, the case fatality rate, the total number of cases and the total

Table 3

SVEIQR homogeneous population model estimates of the prevalence of infectives, prevalence of immunity and inter-epidemic period in a population of 10,000 head

\begin{tabular}{lccccc}
\hline Parameter & Min & $<95 \%$ & Mean & $>95 \%$ & Max \\
\hline$R_{\mathrm{o}}$ & 2.72 & 3.38 & 4.10 & 4.74 & 5.20 \\
Prevalence of infectives $^{\mathrm{a}}$ & 0.96 & 1.54 & 2.20 & 2.91 & 3.50 \\
Prevalence of sequestra $^{\mathrm{a}}$ & 2.19 & 2.62 & 3.70 & 4.50 & 4.96 \\
Prevalence of infection $^{\mathrm{a}}$ & 3.15 & 4.28 & 5.90 & 7.35 & 8.21 \\
Prevalence of immunity $^{\mathrm{a}}$ & 56.7 & 62.7 & 66.8 & 71.5 & 73.2 \\
Inter-epidemic period $^{\mathrm{b}}$ & 2.1 & 2.4 & 3.2 & 4.3 & 4.9 \\
\hline
\end{tabular}

\footnotetext{
a Percent $(\%)$ of animals out of the total population.
}

b Years in the absence of seasonal forcing. 
Table 4

Sensitivity analysis on the impact of the size of the stochastic comparison and seasonality on the persistence of CBPP infection in the SVEIQR homogeneous population model

\begin{tabular}{clllll}
\hline Herd size (head) & \multicolumn{2}{l}{ Stochastic threshold of $0^{\mathrm{a}}$} & & \multicolumn{2}{l}{ Stochastic threshold of $5^{\mathrm{a}}$} \\
\cline { 2 - 3 } \cline { 5 - 5 } & No. of seasonality & 0.5 of seasonality & & No. of seasonality & 0.5 of seasonality \\
\hline 1000 & $96.2^{\mathrm{c}}$ & 93.6 & 54.5 & 46.4 \\
500 & 66.4 & 64.0 & 13.4 & 10.6 \\
400 & 55.2 & 42.2 & 5.0 & 5.4 \\
300 & 37.2 & 25.2 & 3.0 & 1.0 \\
200 & 14.4 & 9.2 & 0.6 & 0.2 \\
100 & 2.0 & 0.4 & 0.4 & 0 \\
75 & 0 & 0 & 0.2 & 0 \\
50 & 0 & 0 & 0 & 0 \\
\hline
\end{tabular}

${ }^{\text {a }}$ When the predict number of transition is below the stochastic threshold comparisons against random numbers are used to determine the number of transitions between the susceptible and exposed compartments. A threshold of 0 turns off this stochastic component of the model. When the threshold is five, all predicted transitions of five or less are compared against a random number and either five or no transitions take place during the time step based on the results of the comparison.

b The amplitude of seasonal forcing was set to 0.5 .

${ }^{c}$ Final herd prevalence- the percent of herds that had at least one infected (infectious or persistently infected) animal at the end of the 6-year modelling period.

mortality. In single isolated herds of 500 head of cattle, a $50 \%$ reduction in the length of the infectious period caused fade-out in essentially $100 \%$ of herds, a $60 \%$ decline in the number of cases and a $73 \%$ decline in mortality.

The results of the three vaccination regimes at the two levels of vaccine efficacy are presented in Table 6 . Vaccination reduced the percentage of herds persistently infected by $53-81 \%$ depending on the regime tested, but did not eliminate infection from all herds. The most effective programme, 3 years of biannual campaigns with a vaccine of $60-80 \%$ efficacy, reduced the percent of persistently infected herds from 10.6 to $2 \%$. Vaccination reduced average total mortality from 111 with no vaccination to between 62 and 41 head lost depending on the programme implemented. A 5-year annual immunization programme with a vaccine having an efficacy of between 50 and $80 \%$ reduced death losses by $44 \%$.

Table 5

SVEIQR homogeneous population model predictions of the effect of reducing the duration of the CBPP infectious period on the persistence of CBPP infection in the model

\begin{tabular}{lllllll}
\hline $\begin{array}{l}\text { Recovery } \\
\text { rate }(\alpha)\end{array}$ & Mean $R_{0}$ & $\begin{array}{l}\text { Final herd } \\
\text { prevalence }(\%)^{\mathrm{a}}\end{array}$ & $\begin{array}{l}\text { Mean duration } \\
\text { infection }^{\mathrm{b}}\end{array}$ & $\begin{array}{l}\text { Case fatality } \\
\text { rate }\end{array}$ & $\begin{array}{l}\text { Mean total } \\
\text { cases }\end{array}$ & $\begin{array}{l}\text { Mean total } \\
\text { mortality }\end{array}$ \\
\hline 56 & 4.10 & 10.6 & 1288 & 33.5 & 331 & 111 \\
42 & 3.36 & 6.4 & 1160 & 28.5 & 256 & 73 \\
28 & 2.37 & 0.2 & 845 & 21.9 & 132 & 29 \\
\hline
\end{tabular}

\footnotetext{
${ }^{a}$ The percent of herds that had at least one infected (infectious or persistently infected) animal at the end of the 6-year modelling period.

b The mean number of days that the disease persisted in the herds modelled in the 500 iterations. Note that in herds where the disease did not fade-out, the duration was truncated at 2141 days ( $\sim 6$ years).
} 
Table 6

Model predictions of the effect of vaccination on the persistence of herd CBPP infection and CBPP-induced mortality

\begin{tabular}{|c|c|c|c|c|}
\hline $\begin{array}{l}\text { Vaccination } \\
\text { programme }^{\mathrm{a}}\end{array}$ & $\begin{array}{l}\text { Vaccine } \\
\text { efficacy }(\varepsilon)\end{array}$ & $\begin{array}{l}\text { Final herd } \\
\text { prevalence }(\%)^{\mathrm{b}}\end{array}$ & $\begin{array}{l}\text { Mean duration } \\
\text { of infection }^{c}\end{array}$ & $\begin{array}{l}\text { Mean total } \\
\text { mortality }\end{array}$ \\
\hline No vaccination & - & 10.6 & 1288 & 111 \\
\hline Annual, 5 years & $.5-.8$ & 4.4 & 1085 & 62 \\
\hline Biannual, 2 years & & 5.0 & 936 & 51 \\
\hline Biannual, 3 years & & 3.0 & 903 & 47 \\
\hline Annual, 5 years & $.6-.8$ & 3.0 & 1056 & 59 \\
\hline Biannual, 2 years & & 5.0 & 940 & 49 \\
\hline Biannual, 3 years & & 2.0 & 840 & 41 \\
\hline
\end{tabular}

\footnotetext{
${ }^{\text {a }}$ Frequency and duration of programme.

b The percent of herds that had at least one infected (infectious or persistently infected) animal at the end of the 6-year simulation.

c The mean number of days that the disease persisted in the herds modelled in the 500 iterations. Note that in herds where the disease did not fade-out, the duration was truncated at 2141 days ( $~ 6$ years).
}

\section{Discussion}

The literature on CBPP is often contradictory and sometimes contentious on a number of issues. In part, this is related to the insidious nature of CBPP. However, in many cases the discrepancies arise from a lack of rigour in the study design and/or performance. For example, wide ranges are given for incubation period, but many authors do not define the parameter they are attempting to measure. In some cases, herd level exposure in large mobs of cattle is used as the starting point and the time to sero-conversion for all those cattle that eventually become ill is taken as the end-point (Turner, 1954). No means were available to document the point of effective contact and many of the cases are undoubtedly tertiary or higher in the chain of transmission. The very long incubation periods occasionally reported in the literature may simply be artefacts of inexact measurement procedures.

Models are a useful approach to consolidating and critically evaluating the range of information available on CBPP. The process of mathematically defining relationships between states and estimating parameters requires one to make clear choices and develop a logically consistent synthesis of information. The output of the model can be compared against what is observed in the field to test how well the synthesis of information in fact reflects the behaviour of the disease. If the undertaking is successful, the result is a powerful tool to help evaluate control options.

The homogeneous SVEIQR model makes full use of quantitative data from laboratorybased epidemiology and is grounded in the knowledge of the participants who are the potential beneficiaries of proposed interventions. The socio-economic dimensions of livelihoods have tremendous impact on the appropriateness of interventions. As both qualitative and quantitative elements are explicitly addressed, this study offers great realism and potential benefit.

The prevalences of infection predicted by the model (3.15-8.21\%) compare favourably with levels detected in large sero-surveys completed in pastoral areas. Zessin et al. (1985) 
found prevalences of $8.1 \%$ in Dinka cattle and $9.2 \%$ in Fallata cattle. A cross-sectional survey of Tuic Dinka cattle conducted during January of 1994 found a prevalence of $8.1 \%$ (McDermott et al., 1987). The seroprevalence of infection in the sample of Jie and Murle cattle collected in the Boma area in March of 2003 was 4.7\% (66/1402). The variation in observed prevalence levels can be accounted for by differences in host population factors such as herd size, breed susceptibility, social exchange practices, seasonal herding patterns and contact structure. These factors vary widely between pastoral communities in East Africa.

It is now known that East Africa shares a common genetic lineage of CBPP mycoplasmas (Lorenzon et al., 2003) and this suggests that regional CBPP strains share some characteristics. Thus, the model should be regionally applicable as far as the biological characteristics of the agent are concerned. However, caution should be exercised when applying the model to new communities due to the variation in host factors described above.

The current formulation of the model was based on estimates of the basic reproduction number derived from serological studies of pastoral groups in Southern Sudan. They manage their livestock in moderate to large composite herds, practice extensive social exchange of cattle, range 100-200 km in their annual migration and have contact with multiple communities in route. It would be best to validate the model and derive estimates of $R_{0}$ in communities outside of southern Sudan using estimates of the average age of seroconversion.

All the pastoral groups of Sudan and the Tatuga of Tanzania reported a pronounced seasonality in clinical cases with the peaks tending to coincide with the very late dry season or the onset of the rains. Environment and weather may play a role (Provost et al., 1987) through variations in conditions that favour survival of the organism or exacerbate clinical disease and shedding of infection. On the other hand, the Tatuga attributed seasonality to higher contact rates in the dry season grazing areas where herds from many areas congregate. This observation was support by the fact that the Kurya of Tanzania, the only sedentary pastoral group studied, did not recognize any seasonality.

This single-herd model provides optimistic estimates that negate inter-herd effects. In its present form it has been useful as a tool to obtain insights into the trends in efficacy of control options. However, herds in pastoral areas are never closed. Cattle movement over long distances is the key strategy that makes livestock husbandry feasible in the dynamic ecosystems of arid and semi-arid areas. Stock theft is a major component of local conflict in pastoral societies throughout the remote areas of East Africa and raided stock are often disbursed to a large number of households. Livestock exchange through bride wealth and loans is an important part of the coping strategies of pastoral livelihood systems and is both a means of spreading risk and the foundation of community institutions. A spatially heterogeneous population model that utilized the SVEIQR model as the basic building block of analysis has been developed from this initial study. The heterogeneous model addresses inter-herd effects and is the subject of a companion paper (Mariner et al., 2006).

The effect of the size of stochastic comparison for transitions from the susceptible to exposed state on the persistence of CBPP in herd sizes of less than 1000 head illustrates the difficulty in estimating the critical community size from models. Addition of the stochastic comparison is more realistic, but the choice of five for herds of 500 animals is a matter of 
expert opinion. Increasing the stochastic comparison beyond five would have further reduced persistence and increased the estimate of the critical community size required to sustain infection indefinitely. For these reasons, the model should be interpreted in terms of principal and relative effects, rather than as a source of absolute estimates of effect.

In the core model with large population size, the total number of cases and persistence of infection in the population is highly sensitive to the CBPP-specific mortality rate and moderately sensitive to the effective contact rate. Theoretically, high mortality rates reduce the infectious period (Eq. (12)) and the basic reproduction number. The implementation of stamping-out procedures is analogous to increasing the CBPP-specific mortality rate in this model. Movement control reduces effective contact rates and the effective reproduction number. Thus, the model supports the conventional wisdom that stamping out and movement control are highly effective weapons in the control of CBPP, where such procedures are acceptable (Hammond and Branagan, 1965).

Although studies on the efficacy of the T1/44 vaccine are numerous, interpretation of results in terms of protection against disease or transmission is fraught with difficulty. The system of Hudson and Turner scores (Hudson and Turner, 1963) utilized in CBPP vaccine trials is meant to standardize scores across different challenge experiences. As controls exhibit more severe signs of infection, the percent protection calculated by these methods is usually greater than the percent of vaccinates that actually remain free of disease. For example, in the 6 month post-vaccination experiment reported by Gilbert et al. (1970) 3 of 16 vaccinates $(18.8 \%)$ exhibited lesions and 2 of $16(12.5 \%)$ had CFT titres yet the $E_{\mathrm{p}}$ score was $94.5 \%$. In the case of a 2 -year post-vaccination experiment 5 of 16 vaccinates $(31.3 \%)$ possessed infected sequestra but the $E_{\mathrm{p}}$ score was $80.0 \%$. These problems are further compounded by the lack of statistical methods to analyze the scores and derive confidence intervals. This suggests a 'numerical bias' is operating where a number, any number, is somehow convincing.

In the analysis described in this paper, conservative estimates of the vaccine's efficacy were utilized (50-80\%). These levels were more in line with the actual prevalence of lesions and other indicators of infection found in challenged vaccinates as described in the vaccine efficacy literature. More importantly, they were more in line with the expert opinion of livestock owners and field veterinary staff (Mariner et al., 2003). The estimates also reflected the documented isolations of $M$. mycoides from the mucosal surfaces and lungs of vaccinates and controls in the absence of other convincing evidence of infection (Davies et al., 1968; Gilbert et al., 1970).

Vaccination in a single isolated herd resulted in a major reduction in mortality, but even under these conditions of perfect quarantine vaccination was not successful in eradicating disease in $4.4 \%$ of herds after 5 years of annual vaccination. If the stochastic comparison is removed, the impact of vaccination on the persistence of infection is markedly poorer. This suggests that although vaccination can result in important reductions in disease losses, a disease eradication programme based on vaccination and quasi-movement control in pastoral areas will not succeed. In order for eradication based on vaccination alone to succeed, significant improvements in vaccine efficacy, duration of immunity and safety are required.

Effective treatment of disease reduces transmission and the effective reproduction number by decreasing the duration of infection and the infectiousness of cases. Controlled 
studies of the effect of treatment of CBPP on the clinical course of disease have not been described. On the other hand, treatment of clinical cases is now standard field practice and livestock owners, community animal health workers (CAHW) and field veterinarians attest to its value. The potential impact of reducing the infectious period on disease persistence and total mortality (Table 5) was in the same range as the impact of currently available vaccines (Table 6). This would suggest that research to document the efficacy of prudent and feasible treatment regimes should warrant the same attention as vaccine development. A documented treatment alternative would have the added advantage of providing a CBPP control mechanism that would be driven by private interests and essentially self-financing.

Considering the current financial constraints on public sector services and the epidemiological likelihood that CBPP control would be a costly and open-ended commitment, it is in the interests of national authorities, the veterinary professional and the livestock owners to seek sustainable, low-cost models for CBPP control. It is noteworthy that African pastoral communities practiced a form of effective traditional CBPP vaccination prior to the advent of vaccination in Europe and colonization (Blancou, 1996). Direct involvement of community animal health workers in the provision of CBPP vaccination under the supervision of public or private veterinarians offers the best hope of building a control programme that meets the public's needs.

At present, access to CBPP vaccines is heavily restricted in most countries and in reality only available from sporadic vaccination campaigns undertaken by the public veterinary services. The involvement of private and community-based service providers in elective vaccination programmes would require a policy decision on the part of national governments and the liberalization of access to regulated supplies of CBPP vaccine through appropriately licensed commercial channels.

\section{Acknowledgement}

The authors would like to acknowledge the support of the Community-based Animal Health and Participatory Epidemiology (CAPE) Unit, Pan African Programme for the Control of Epizootics (PACE), African Union/Interafrican Bureau for Animal Resources for their support of the fieldwork, initial modelling work and preparation of manuscripts. The CAPE Unit is funded by the Department for International Development (United Kingdom). The authors wish to thank the European Union, the PACE Epidemiology Unit and the Animal Health Division of FAO for their support of the main modelling study.

\section{References}

Anderson, R.M., May, R.M., 1991. Infectious Disease of Humans. Oxford University Press, New York.

Blancou, J., 1996. Early methods of surveillance and control for contagious bovine pleuropneumonia. Rev. Sci. Tech. 15, 1241-1282.

Bolske, G., Msami, H.M., Gunnarsson, A., Kapaga, A.M., Loomu, P.M., 1995. Contagious bovine pleuropneumonia in northern Tanzania, culture confirmation and serological studies. Trop. Anim. Health Prod. 27, 193-201. 
Bygrave, A.C., Moulton, J.E., Shifrine, M., 1968. Clinical, serological and pathological findings in an outbreak of contagious bovine pleuropneumonia. Bull. Epizoot. Dis. Afr. 16, 21-46.

Campbell, A.D., Turner, A.W., 1953. Studies of contagious bovine pleuropneumonia of cattle. IV. An improved complement fixation test. Aust. Vet. J. 29, 154-163.

Catley, A., 1999. Participatory Approaches to Veterinary Epidemiology: Methods on the Move. Sustainable Agriculture and Rural Livelihoods Programme, IIED, London, 99 pp.

Davies, G., Masiga, W.N., Shifrine, M., Read, W.C.S., 1968. The efficacy of $\mathrm{T}_{1}$ strain broth vaccine against contagious bovine pleuropneumonia: Preliminary in-contact trials. Vet. Rec. 83, 239-244.

Diekmann, O., Heesterbeek, J.A.P., 2000. Mathematical Epidemiology of Infectious Diseases: Model Building, Analysis and Interpretation. John Wiley \& Sons, Chichester.

Dietz, K., 1975. Transmission and control of arboviral diseases. In: Ludwig, D., Cooke, K.L. (Eds.), Epidemiology. SIAM, Philadelphia, pp. 104-121.

Gilbert, F.R., Davies, G., Read, W.C., Turner, G.R., 1970. The efficacy of $\mathrm{T}_{1}$ strain broth vaccine against contagious bovine pleuropneumonia: in-contact trials carried out six and twelve months after primary vaccination. Vet. Rec. 86, 29-33.

Gourlay, R.N., 1965. Comparison between some diagnostic tests for contagious bovine pleuropneumonia. J. Comparat. Pathol. 75, 97-109.

Grenfell, B.T., Kleczkowski, A., Gilligan, C.A., Bolker, B.M., 1995. Spatial heterogeneity, nonlinear dynamics and chaos in infectious diseases. Stat. Methods Med. Res. 4, 160-183.

Hammond, J.A., Branagan, D., 1965. Contagious bovine pleuropneumonia in Tanganyika. Bull. Epizoot. Dis. Afr. $13,121-147$.

Huddart, J.E., 1960. Bovine contagious pleuropneumonia - a new approach to field control in Kenya. Vet. Rec. 72 , 1253-1254.

Hudson, J.R., Turner, A.W., 1963. Contagious bovine pleuropneumonia: a comparison of the efficacy of two types of vaccine. Aust. Vet. J. 39, 373-385.

IAEA, 1992. The Sero-Monitoring of Rinderpest Throughout Africa. Phase II. FAO/IAEA Division of Nuclear Techniques in Food and Agriculture, International Atomic Energy Agency, Vienna.

IAEA, 1993. The Sero-Monitoring of Rinderpest Throughout Africa. Phase II. FAO/IAEA Division of Nuclear Techniques in Food and Agriculture, International Atomic Energy Agency, Vienna.

Karst, O., 1971. A comparison of 2 vaccines against contagious bovine pleuropneumonia. Res. Vet. Sci. 12, $18-22$.

Le Goff, C., Thiaucourt, F., 1998. A competitive ELISA for the specific diagnosis of contagious bovine pleuropneumonia (CBPP). Vet. Microbiol. 60, 179-191.

Lesnoff, M., Laval, G., Chalvet-Monfrey, K., Lancelot, R., Thiaucourt, F., 2004. A mathematical model of the effects of chronic carriers on the within-herd spread of contagious bovine pleuropneumonia in an African mixed crop-livestock system. Prev. Vet. Med. 62, 101-117.

Lorenzon, S., Arzul, I., Peyraud, A., Hendrikx, F., Thiaucourt, F., 2003. Molecular epidemiology of contagious bovine pleuropneumonia by multilocus sequence analysis of Mycoplasma mycoides subspecies mycoides biotype SC strains. Vet. Microbiol. 93, 319-333.

Majok, A.A., Zessin, K.H., Baumann, M.P.O., Farver, T.B., 1991. Analyses of baseline survey data on rinderpest in Bahr el Ghazal Province, with proposal of an improved vaccination strategy against rinderpest for southern Sudan. Trop. Anim. Health Product. 23, 186-196.

Mariner, J.C., Araba, A., Makungu S., 2003. Consultancy on the Dynamics of CBPP Endemism and the Development of Effective Control/Eradication Strategies for Pastoral Communities: Final Data Collection Report. The Community Animal Health and Participatory Epidemiology Unit of the African Union InterAfrican Bureau for Animal Resources, Nairobi.

Mariner, J.C., McDermott, J.J., Heesterbeek, J.A.P., Thomson, G., Roeder, P.L., Martin, S.W., 2006. A spatially heterogeneous population model for contagious bovine pleuropneumonia transmission and control in pastoral communities of East Africa. Prev. Vet. Med. 73, 75-91.

Mariner, J.C., Paskin, R., 2000. Manual on Participatory Epidemiology. Food and Agriculture Organisation, Rome, $81 \mathrm{pp}$.

Masiga, W.N., Domenech, J., 1995. Overview and epidemiology of contagious bovine pleuropneumonia in Africa. Rev. Sci. Tech. 14, 611-630. 
Masiga, W.N., Domenech, J., Windsor, R.S., 1996. Manifestation and epidemiology of contagious bovine pleuropneumonia in Africa. Rev. Sci. Tech. 15, 1283-1308.

Masiga, W.N., Read, W.C.S., 1972. Comparative susceptibility of Bos indicus and Bos taurus to contagious bovine pleuropneumonia, and the efficacy of the T1 broth culture vaccine. Vet. Rec. 90, 499-502.

Masiga, W.N., Rurangirwa, F.R., Roberts, D.H., Kakoma, I., 1978. Contagious bovine pleuropneumonia: comparative efficacy trial of the (freeze-dried French T1 vaccine) and the T1 broth culture vaccine (Muguga). Bull. Anim. Health Prod. Afr. 26, 216-223.

McDermott, J.J., Deng, K.A., Jayatileka, T.N., El Jack, M.A., 1987. A cross-sectional cattle disease study in Kongor Rural Council, southern Sudan. I. Prevalence estimates and age, sex and breed associations for brucellosis and contagious bovine pleuropneumonia. Prev. Vet. Med. 5, 111-123.

Mefit-Babtie, S., 1983. Development Studies in the Jonglei Canal Area. Final Report, vol. 4. Livestock Studies. Government of the Democratic Republic of Sudan, Khartoum.

Niamir-Fuller, M., 1999. A review of recent literature on pastoralism and transhumance in Africa. In: NiamirFuller, M. (Ed.), Managing Mobility in African Rangelands. Intermediate Technology Publications, London, pp. $18-46$.

Parker, A.M., 1960. Contagious bovine pleuropneumonia. Production of complement-fixing antigen and some observations on its use. Bull. Epiz. Dis. Afr. 8, 111-119.

Provost, A., Perreau, P., Breard, A., Le Goff, C., Martel, J.L., Cottew, G.S., 1987. Contagious bovine pleuropneumonia. Rev. Sci. Tech. 6, 625-679.

Schneider, H.P., van der Lugt, J.J., Hubschle, O.J.B., 1994. Contagious bovine pleuropneumonia. In: Coetzer, J.A.W., Thomson, G.R., Tustin, R.C. (Eds.), Infectious Disease of Livestock with Special Reference to Southern Africa, vol. 2. Oxford University Press, New York.

Stone, S.S., Bygrave, A.C., 1969. Contagious bovine pleuropneumonia: comparison of serological tests and postmortem observations in cattle with resolving lung lesions. Bull. Epizoot. Dis. Afr. 17, 11-19.

Thiaucourt, F., Yaya, A., Wesonga, H., Huebschle, O.J., Tulasne, J.J., Provost, A., 2000. Contagious bovine pleuropneumonia. A reassessment of the efficacy of vaccines used in Africa. Ann. N.Y. Acad. Sci. 916, 71-80.

Turner, A.W., 1954. Epidemiological characteristics of bovine contagious pleuropneumonia. Aust. Vet. J. 312 317.

Turner, A.W., 1959. Pleuropneumonia Group of Diseases. In: Stableforth, A.W., Galloway, I.A. (Eds.), Infectious Diseases of Animals, Disease Due to Bacteria, vol. 2. Butterworths Science Publications, London, pp. 437 463.

Turner, A.W., Campbell, A.D., 1937. Studies on contagious bovine pleuropneumonia of cattle. IV. An improved compliment fixation test. Aust. Vet. J. 13, 183-186.

Turner, A.W., Etheridge, J.R., 1963. Slide agglutination tests in the diagnosis of contagious bovine pleuropneumonia. Aust. Vet. J. 39, 445-451.

Wesonga, H.O., Thiaucourt, F., 2000. Experimental studies on the efficacy of T1sr and T1/44 vaccine of Mycoplasma mycoides subspecies mycoides (small colony) against a field isolate causing contagious bovine pleuropneumonia in Kenya-effect of a revaccination. Revue Elev. Med. Vet. Pays Trop. 53, 313-318.

Windsor, R.S., 2000. The eradication of contagious bovine pleuropneumonia from south western Africa: a plan for action. Ann. N.Y. Acad. Sci. 916, 326-332.

Windsor, R.S., Masiga, W.N., 1977. Investigations into the role of carrier animals in the spread of contagious bovine pleuropneumonia. Res. Vet. Sci. 23, 224-229.

Windsor, R.S., Masiga, W.N., Read, W.C., 1972. The efficacy of $\mathrm{T}_{1}$ strain broth vaccine against contagious bovine pleuropneumonia: in-contact trials carried out two years after primary vaccination. Vet. Rec. 90, 2-5.

Zessin, K.H., Baumann, M., Schwabe, C.W., Thorburn, M., 1985. Analysis of baseline surveillance data on contagious bovine pleuropneumonia in the Southern Sudan. Prev. Vet. Med. 3, 371-389. 